\title{
Comparison of phase slippage processes in Josephson junctions and in charge density wave stacked junctions
}

\author{
Yu.I. Latyshev \\ Kotelnikov Institute of Radio-Engineering and Electronics, RAS, 11-7 Mokhovaya Str., Moscow 125009, Russia \\ Email: lat@cplire.ru
}

Received December 28, 2011

\begin{abstract}
We discuss time-space symmetry in the processes of phase slippage in Josephson junctions and charge density wave (CDW) stacked junctions. In Josephson junctions phase slip by $2 \pi$ occurs periodically in time with a period $T$ above critical current with a frequency obeys Josephson relation $v=1 / T=2 \mathrm{eV} / \mathrm{h}$, where $V$ is the voltage on the junction. In CDW stacks at voltage on the stack above threshold value the CDW dislocation appears in the weakest junction of the stack. This phase dislocation corresponds to the local phase slippage by $2 \pi$. With voltage increase a new dislocations appear forming periodic array of dislocations with a period $L$. The inverse spacing $1 / L$ follows the analog of Josephson equation $v_{F} / L=2 \mathrm{eV} / \mathrm{h}$.
\end{abstract}

PACS: 75.50.+r Quantum spin liquids, valence bond phases and related phenomena;

71.45.Lr Charge-density-wave systems;

73.40.Gk Tunneling.

Keywords: Josephson junction, phase slippage, CDW stacked junction, CDW phase soliton.

\section{Introduction}

The two condensed electronic states superconducting state and CDW state have some common features. In both cases the condensed state is formed at the Fermi surface and the gap is opened in the electronic spectra. Also the order parameter of condensed state in both cases is characterized by the amplitude and the phase. However, in a case of superconductivity the pairing happens between electrons with total momentum of the pair equals to zero. In a case of the CDW state the pairing happens between electron and hole with total momentum of the pair is $2 p_{F}$ or the wave number is $2 k_{F}$. That means that the CDW condensate is correlated in a space with a wave vector of $2 k_{F}$. Here we consider an analogy with the ac Josephson effect in the CDW stacked junctions.

\section{Experimental}

$\mathrm{NbSe}_{3}$ is widely known as a typical layered CDW material with two Peierls transitions at $T_{p 1}=145 \mathrm{~K}$ and $T_{p 2}=59 \mathrm{~K}$. A remarkable feature of this compound is a very high interlayer conductivity anisotropy $\sigma_{b c} / \sigma_{a^{*}} \sim 10^{4}$ at low temperatures. Therefore, a transport across the layers occurs due to the interlayer tunneling similar to the layered high temperature superconductors [1] and that can be used for the CDW gap spectroscopy [2]. The stacked junctions usually used for spectroscopy have typically micron scale in-plane sizes and contains few tens of elementary tunnel junctions along the $a^{*}$-axes. Schematically the stacked junction (or mesa) is shown in Fig. 1. We developed double sided focused ion beam technique for mesas fabrication [3].

\section{Results and discussion}

As the most prominent features of the interlayer tunneling spectra there were found the CDW gap peaks for both $\mathrm{CDWs}$ in $\mathrm{NbSe}_{3}$ at the bias voltages $V=2 \Delta_{1,2}[2,4]$

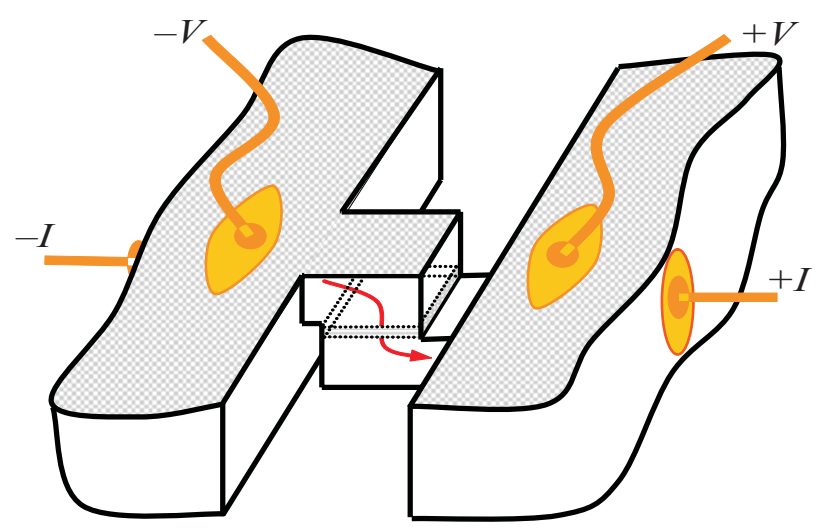

Fig. 1. A schematic view of the mesa type structure. 

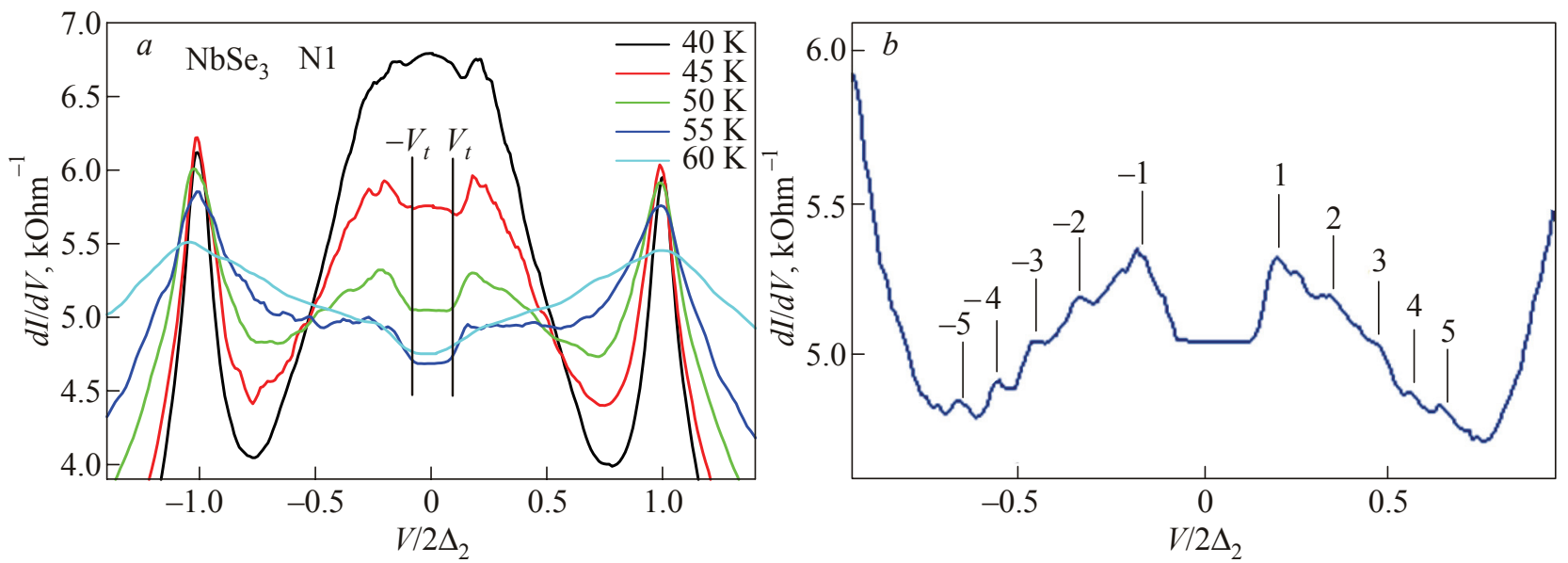

Fig. 2. Interlayer tunneling spectra of $\mathrm{NbSe}_{3}$ mesa at various temperatures below lower Peierls transition temperature $T_{p 2}(a)$ and in more details at $T=50 \mathrm{~K}(b)$. The bias voltage is normalized to the value of CDW gap $2 \Delta_{2}=60 \mathrm{mV}$. Note the threshold feature at $V=V_{t}$ and quasi-periodic fine structure at $V>V_{t}$ symmetric for positive and negative $V$.

(Fig. 2,a). Another remarkable feature is a threshold behavior of interlayer tunneling conductivity at bias voltage $V_{t}$ much less than $2 \Delta$. That feature has been interpreted as a voltage necessary for a formation of the CDW phase dislocation (or $2 \pi$-phase soliton) in the weakest junction of the stack [5]. The phase soliton is schematically shown in Fig. 3 as a pink region that is localized within several CDW units along the chain. It contains excess CDW period and thus has a charge $2 e$ (or excess phase of $2 \pi$ ). The presence of charge soliton breaks locally transversal coherence of the junction as shown in a Fig 3. In other words that means a local phase slippage between adjacent chains.

The threshold voltage for the formation of CDW phase soliton in some sense is similar to the critical current $I_{c}$ in a Josephson junction since at $I>I_{c}$ the stationary phase distribution across the junction becomes non-stationary and

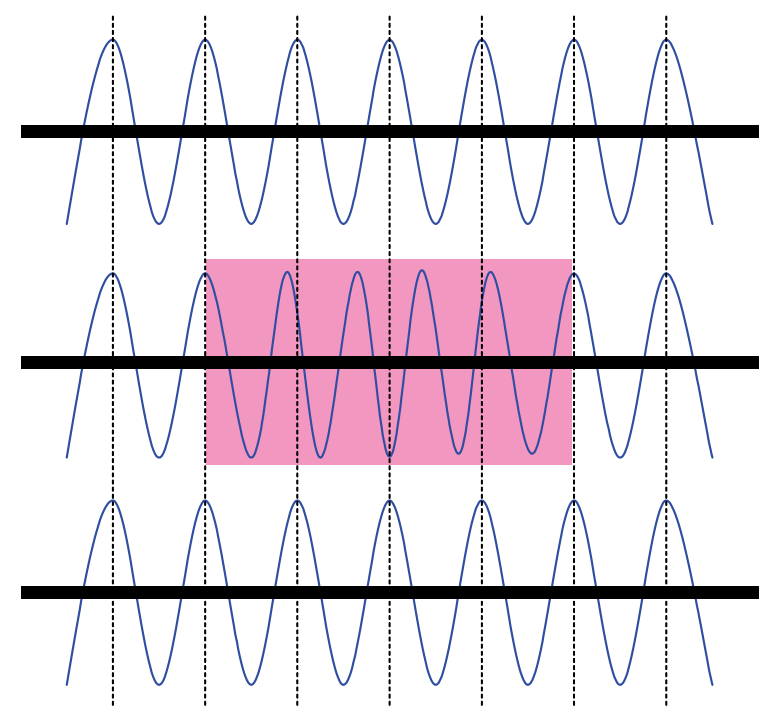

Fig. 3. Schematic view of $2 \pi$-soliton formation in the weakest CDW coupled junction. fluxons starts to move along the junction to release the critical phase difference by phase slippage [6].

That was also found that a threshold voltage feature is accompanied by fine quasiperiodic structure of the steps on $d I / d V(V)$ [5]. This structure is seen in Fig. 2,b and much more clearly in the second derivative picture (Fig. 4) after subtraction of the monotonic background. The picture is well symmetric for positive and negative bias voltages on the junction. The voltage of appearance the second step $V_{2}-V_{t}$ is close to $V_{t}$ and then gradually decreases for $V_{n}-V_{n-1}$. We, therefore, consider that the step structure is associated with the formation of the 2nd, 3rd and so on dislocations in the junction as it is shown in the bottom panel of Fig. 4.

The appearance of one dislocation reduces the energy for creation the next one. That can explain the reduction of peaks periodicity with $n$. As far as dislocations are charged each of them bearing elementary charge $2 e$ they experience Coulomb repulsion. That should result in a periodic array

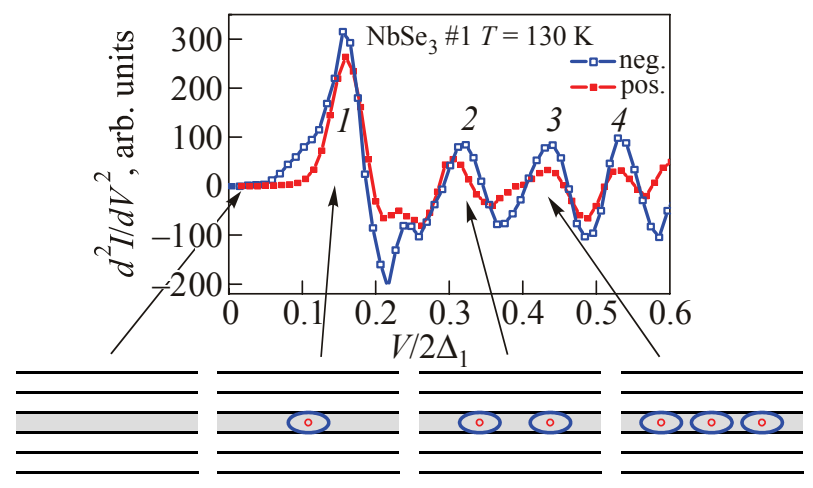

Fig. 4. A derivative of interlayer tunneling spectrum of the $\mathrm{NbSe}_{3}$ stack junction at $50 \mathrm{~K}$ for positive $(\square)$ and negative $(\square)$ polarity of the bias voltage. The bottom panel schematically shows that each maximum of $d^{2} I / d V^{2}$ is accompanied by the formation in a junction a new phase dislocation marked in a picture as an ellipse. 


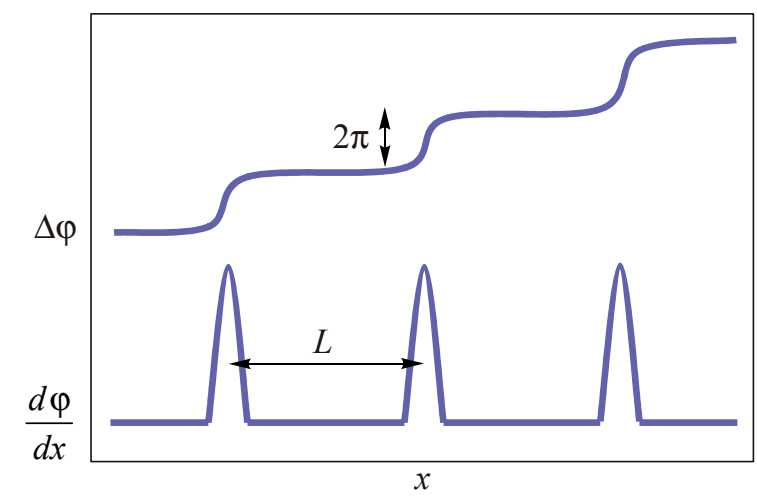

Fig. 5. Spatial variation of the phase $\Delta \varphi$ and phase derivative $d \varphi / d x$ along the phase dislocation array in CDW coupled junction.

(1D lattice) of phase CDW solitons in the weakest junction. Figure 5 shows a plot of the phase difference $\Delta \varphi$ along the junction containing dilute lattice of CDW phase solitons. The steps of phase difference by $2 \pi$ in Fig. 5 correspond to positions of phase solitons (CDW dislocations).

The lower panel shows dependence of $d \varphi / d x(x)$. Both pictures show the space periodicity, $L$, between phase slippage events. A theory of phase decoupling induced by voltage $V$ on the junction [5,7] gives the relationship between the $V$ and $L$

$$
2 e V=h v_{F} d j / d x
$$

where $h$ is Plank constant and $v_{F}$ is Fermi velocity. From Eq. (1) it follows that $V$ is proportional to $1 / L: 1 / L=$ $=2 e V /\left(h v_{F}\right)$. For a value of $V \approx \Delta$ that provides the spacing value of $L=200-300 \mathrm{~nm}$. The remarkable point here is that Eq. (1) looks very similar to the ac Josephson equation for the phase slippage in Josephson junction at $I>>I_{C}$ if one replaces $v_{F} d j / d x$ by $d \varphi / d t$ :

$$
2 e V=h d \varphi / d t .
$$

We can then plot a very similar to Fig. 5 picture for the phase difference increase in Josephson tunnel junction in time (Fig. 6). Here the phase difference by $2 \pi$ happens with time periodicity $T$. Remarkable that $T$ is inversely proportional to the voltage on the junction: $1 / T=2 \mathrm{eV} / \mathrm{h}$ in a similar way as $L$ is inversely proportional to the voltage $V$ on the CDW coupled junction. Our consideration shows that phase slippage in space in the CDW coupled junctions behaves exactly in the same way as the phase slippage in time in the Josephson (superconducting phase coupled) junction.

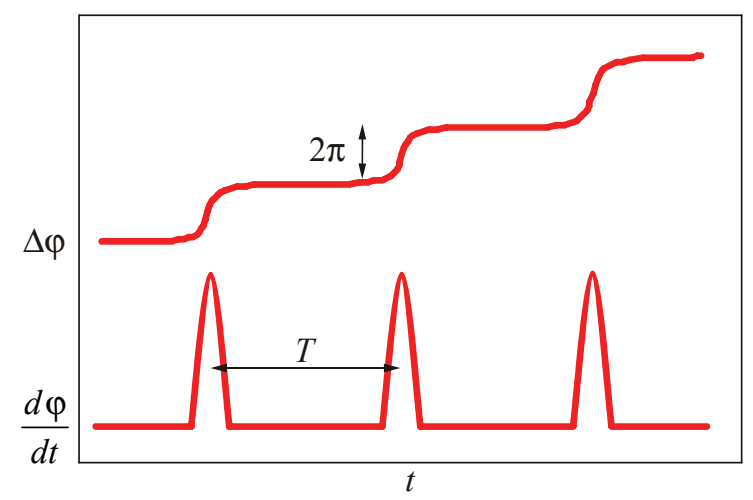

Fig. 6. Time variation of the phase $\Delta \varphi$ and phase time derivative $d \varphi / d t$ in Josephson junction.

\section{Conclusions}

The observed space-time symmetry in the phase slippage processes of CDW and superconducting phase coupled junctions seems to reflect the general space-time symmetry in formation of superconducting and CDW condensates. In superconducting condensate Cooper pairs are formed by electrons with opposite momenta. They are correlated in time within a time domain of $2 \Delta / h$, while in the CDW condensate the electron-hole pairs are correlated in space with the wave number $2 k_{F}$.

Note also that the observed symmetry of the ac Josephson effect in superconducting and CDW phase coupled junctions may be useful for some future Josephson applications in the CDW systems.

The author is highly acknowledged to S.A. Brazovskii for fruitful discussions. The work has been supported by RFBR grant No 11-02-01379-a and RAS programs.

1. V.M. Krasnov, A. Yurgens, D. Winkler, P. Delsing, and T. Claeson, Phys. Rev. Lett. 84, 5860 (2000).

2. Yu.I. Latyshev, P. Monceau, A.A. Sinchenko, L.N. Bulaevskii, S.A. Brazovskii, T. Kawae, and T. Yamashita, J. Phys. A: Math. Gen. 36, 9323 (2003).

3. Yu.I. Latyshev, S.-J. Kim, and T. Yamashita, IEEE Trans. Appl. Supercond. 9, 4312 (1999).

4. A.P. Orlov, Yu.I. Latyshev, A.M. Smolovich, and P. Monceau, JETP Lett. 84, 89 (2006).

5. Yu.I. Latyshev, P. Monceau, S. Brazovskii, A.P. Orlov, and T. Fournier, Phys. Rev. Lett. 96, 116402 (2006).

6. A. Barone and G. Paterno, Physics and Applications of the Josephson Effect, New York: John Wiley \& Sons (1982).

7. S. Brazovskii and S.A. Matveenko, Phys. Rev. B77, 155432 (2008). 